\title{
Cumplimiento de los Protocolos de Prevención de la Legionelosis en Edificios Públicos
}

\section{Legionnaires' disease prevention protocol performance in public buildings}

\author{
Enrique Gea-Izquierdo
}

Universidad de Málaga, España. enriquegea@telefonica.net, egea@uma.es

Recibido 18 Junio 2008/Enviado para Modificación 21 Diciembre 2008/Aceptado 28 Diciembre 2008

\section{RESUMEN}

Objetivo Investigar la relación entre el cumplimiento del Real Decreto 865/2003 y el Decreto 287/2002, por los que se establecen los criterios higiénico-sanitarios para la prevención y control de la legionelosis, y el riesgo debido a un deficiente mantenimiento higiénico- preventivo de las instalaciones de agua.

Métodos Estudio descriptivo realizado en la provincia de Málaga (España) sobre una muestra correspondiente a 214 edificios públicos, 83 variables e interpretación sobre el porcentaje de cumplimiento de la normativa. Medida del riesgo, definición de clusters (tipificación del riesgo), estimadores clásicos y robustos, estimación jackknife e intervalos de confianza bootstrap.

Resultados Riesgo por incumplimiento parcial entre el 11,1 (riego, Centros Privados de ESO) y $85,7 \%$ (AFS, Institutos de Educación Secundaria). La normativa se cumple en 82 edificios, 130 la incumplen y 2 no presentan instalaciones de riesgo. Cumplimiento total $38 \%$. Estimación para el cumplimiento parámetros de localización 0,33-0,36 (33-36 \%) y de escala 0,11-0,16. Estimador jackknife del sesgo reducido 0,35071 (estimación media). Intervalos de confianza bootstrap con extremos [0.26, 0.47].

Conclusión El menor riesgo por incumplimiento parcial corresponde a Institutos de Educación Secundaria y el mayor por distribución a los hoteles de cuatro y tres estrellas. Selección del parámetro de localización estimador de Huber y de escala NMAD. Atendiendo al parámetro «razón poblacional» y estimador "razón muestral», el estimador jackknife del sesgo reducido es insesgado. Intervalos de confianza basados en las técnicas bootstrap, partiendo de la media 0,2- recortada muestral y coeficiente de confianza 0,95 , muy aproximados.

Palabras Clave: Prevención de enfermedades, control, riesgos laborales, legionelosis, instalaciones públicas (fuente: DeCS, BIREME).

\section{ABSTRACT}

Objective Studying the relationship between fulfilment of royal decree 865/2003 and decree 287/2002 (establishing hygienic-sanitary criteria for preventing and 
controlling Legionnaires' disease) and risk arising from deficient preventativehygienic water facility maintenance.

Methods This was a descriptive study carried out in the province of Málaga (Spain) on a sample of 214 public buildings using 83 variables and interpreting legislation fulfilment percentages. Risks were measured, clusters defined (related to risk typification) and classic and robust estimators, jack-knife estimation and bootstrap confidence intervals were calculated.

Results $11.1 \%$ (irrigation, ESO private centres) and $85.7 \%$ (AFS, secondary education institutions) partial non-fulfilment/breach risks were found. Eighty-two buildings complied with current standards, 130 failed to fulfil them and 2 did not display risk facilities. There was $38 \%$ overall compliance with established norms. Location parameter fulfilment was estimated as being 0.33-0.36 (33-36\%) and scale as $0.11-0.16$. Reduced bias jack-knife estimator was 0.35071 (average estimation). Bootstrap confidence interval extremes were 0.26 and 0.47 .

Conclusion Secondary education institutions represented the least risk due to partial noncompliance and four- and three-star hotels the greatest. Huber estimator was selected as location parameter and NMAD as scale parameter. Regarding the population ratio parameter and sample ratio estimator, it was considered that the jack-knife estimator of reduced bias was unbiased. Confidence intervals based on bootstrap techniques (starting from 0.2-trimmed mean sample and a 0.95 confidence coefficient) were very approximate.

Key Words: Prevention, control, risk, legionnaires' disease, public facility (source: $\mathrm{MeSH}, \mathrm{NLM}$ ).

$\mathrm{E}$ 1 objeto existen multitud de planteamientos de estudios epidemiológicos $(1,2)$, así como referidos al tipo de detección del microorganismo $(3,4)$ y medio de cultivo $(5,6)$. Igualmente, se ha relacionado el aumento en la proximidad de la fuente y la frecuencia de exposición con el riesgo de contagio (7). En algunos sistemas la vigilancia continuada, la definición de estrategias centradas y su puesta en práctica, pueden mostrar resultados positivos para la prevención de la legionelosis (8).

El estudio de la incidencia de la legionelosis y las consecuencias que sobre la población pudiera tener es bastante conocido. Por el contrario, se formula la necesidad de contrastar el cumplimiento de la normativa $(9,10)$ y su aplicación en los diferentes sistemas de intercambio aire-agua. En el estudio se examina la influencia de la aplicación de los métodos preventivos y la posibilidad del riesgo de desarrollo de Legionella pneumophila. Se consideran distintos tipos de edificios públicos, riesgo presente y tipo de instalación o sistema. El conocimiento de la aplicación real de la normativa podrá ser considerado como indicativo del grado de implementación de medidas preventivas frente a la legionelosis en el área de estudio. 


\section{MÉTODOS}

Muestra de estudio

Se selecciona una muestra de 214 edificios de pública concurrencia (Tabla 1) de la provincia de Málaga (España). La información se recogió en los meses comprendidos entre octubre y diciembre del año 2005 mediante cuestionario al Director/Gerente del Edificio y visita a los edificios representativos. La tasa de respuesta del estudio fue del $21,5 \%$. El tipo y número de edificios son: Hospitales/ 4, Administración (no Junta de Andalucía)/6, Junta de Andalucía/5, Hotel 2 estrellas/21, Hotel 3 estrellas/23, Hotel 4 estrellas/20, Hotel 5 estrellas/4, Hotel Gran Lujo/4, Instituto de Educación Secundaria (público)/23, Centro Público de Educación Infantil y Primaria/54, Centro Privado de Formación Profesional (FP)/ 12, Centro Privado de Bachillerato/10, Centro Privado de Educación Infantil y Primaria/14 y Centro Privado de Educación Secundaria Obligatoria (ESO)/14. En todos los casos se informó al responsable del edificio objeto del estudio y de las características del mismo.

Tabla 1. Tipo de edificio

\begin{tabular}{lcc}
\hline \multicolumn{1}{c}{ Tipo de edificio } & $\begin{array}{c}\text { Porcentaje } \\
\text { válido }\end{array}$ & $\begin{array}{c}\text { Porcentaje } \\
\text { acumulado }\end{array}$ \\
\hline Hospital & 1,9 & 1,9 \\
Administración (No Junta) & 2,8 & 4,7 \\
Junta de Andalucía & 2,3 & 7,0 \\
Hotel 2 Estrellas & 9,8 & 16,8 \\
Hotel 3 Estrellas & 10,7 & 27,6 \\
Hotel 4 Estrellas & 9,3 & 36,9 \\
Hotel 5 Estrellas & 1,9 & 38,8 \\
Hotel Gran Lujo & 1,9 & 40,7 \\
Instituto de Educación Secundaria & 10,7 & 51,4 \\
Centro Público de Educación Infantil y Primaria & 25,2 & 76,6 \\
Centro Privado de FP & 5,6 & 82,2 \\
Centro Privado de Bachillerato & 4,7 & 86,9 \\
Centro Privado de Educación Infantil y Primaria & 6,5 & 93,5 \\
Centro Privado de Educación Secundaria Obligatoria & 6,5 & 100,0 \\
Total & 100,0 & \\
\hline
\end{tabular}

Método y variables de estudio

En el estudio han sido consideradas 83 variables agrupadas en "14 clusters/ tipos de instalaciones (apartados I- XIV)": agua caliente sanitaria (ACS)/ acumuladores (I), agua fría sanitaria (AFS)/ depósitos de agua (aljibes) (II), torres de refrigeración/condensadores evaporativos/condensadores adiabáticos (III), bañeras (sin recirculación, uso individual) (IV), piscinas (con recirculación, uso colectivo, spas o similar) (V), humectadores/ enfriamiento evaporativo/ otros (VI), líneas de agua en unidades dentales (VII), fuentes ornamentales (VIII), humectación 
en conductos de aire (IX), riego por aspersión (inclusive aerosol) (X), instalaciones termales (XI), acuarios (descubiertos) (XII), sistemas de agua contra incendios (SCI) (XIII) y equipos de terapia respiratoria (XIV).

Las 14 categorías anteriores incluyen variables según la descripción del cluster y otras relativas a: la procedencia del agua, notificación de los equipos, control físico- químico y microbiológico del agua, periodicidad de los análisis (realizados por laboratorios homologados), distancias entre fuentes-personas expuestas, presencia de válvulas de vaciado, separadores de gotas, equipos de desinfección, tipo de desinfección, programa y registro de mantenimiento, cursos de formación de personal, empresas de tratamiento registradas, control de la temperatura, equipos de filtración, seguridad de acceso a las instalaciones, material de la instalación, ubicación y protección de los equipos, acceso al interior, y disponibilidad de ficha de seguridad para los productos químicos que se apliquen.

Según las variables y agrupaciones, se calculan los porcentajes de incumplimiento de la normativa atendiendo al tipo de edificio. Se analiza la distribución del riesgo de desarrollo de la bacteria relacionando el no cumplimiento (\%) con la agrupación (apartado) y tipo de edificio, para observar la concentración máxima del riesgo y tipos de edificios más críticos. Asimismo, se considera el total de edificios independientemente del tipo de que se trate, representando el cumplimiento de la normativa por medio de una gráfica de sectores (número de edificios/ porcentaje sobre el total).

Se estima conveniente la utilización de "Métodos Robustos" con el estudio de estimadores robustos para el parámetro de localización ("media") o de escala ("desviación típica") de la población de donde se ha obtenido la muestra. Es interesante el uso de estimadores robustos que no se vean sumamente afectados por la presencia de datos anómalos.

Con los "Métodos de Remuestreo" se estima no sólo algunas características de la población de donde procede la muestra que se remuestrea (media), sino además características del estimador que se utiliza en tales estimaciones (sesgo) o inclusive su propia distribución en el muestreo. Se determina la estimación jackknife del sesgo de los estimadores del cumplimiento (en porcentaje) medio de la normativa en la provincia de Málaga. Se corrigen las estimaciones, obteniendo los estimadores jackknife de sesgo reducido y presentación de una estimación robusta y con menor sesgo del parámetro de localización en estudio. La muestra del estudio tiene tamaño $n=14$ que proporcionó los datos en porcentajes. 
Mediante el bootstrap se extraen conclusiones de la población de donde proceden los datos, muestreándolos repetidamente. Indicar que mientras que con el jackknife se obtienen siempre los mismos resultados, con esta técnica cada vez que se realiza una estimación bootstrap se puede obtener resultados distintos (al ejecutar la misma función el resultado para los mismos datos puede variar cada vez).

En la determinación del intervalo de confianza de la desviación típica poblacional se utiliza su estimador sustitución (desviación típica muestral) y en el intervalo percentil otro estimador robusto como es la desviación absoluta mediana estandarizada $N M A D$.

Análisis estadístico

Los programas informáticos utilizados en la recopilación e interpretación de datos son respectivamente: SPSS (Copyright SPSS Inc., 1989-2004. Windows. Version 13.0.1.20 Nov 2004) y R Development Core Team(2007), R:A language and environment for statistical computing, R Foundation for Statistical Computing, Vienna, Austria.

\section{RESULTADOS}

El mayor porcentaje de cumplimiento es para el apartado I (Agua caliente sanitaria/ Acumuladores, 99,3\%) y el menor el VII (Líneas de agua en unidades dentales, $0 \%$ ) (Tabla 2).

En aquellos edificios "tipo" en los que se ha implantado alguna medida preventiva frente a Legionella pneumophila pero que resulta insuficiente, el riesgo (valor cumplimiento>0) está repartido. Según el Real Decreto 865/2003 y la clasificación presentada ( $\mathrm{p}$-valor=1,210e-10), de los 214 edificios muestreados 212 disponían de instalaciones que utilizaban agua en su funcionamiento, produciendo aerosoles y con ubicación de las mismas en el interior o exterior del edificio (uso colectivo), pudiendo ser susceptibles de convertirse en focos para la propagación de la legionelosis durante su funcionamiento, pruebas de servicio o mantenimiento. Los apartados I- XIV y los porcentajes de cumplimiento de cada uno presentan algunas diferencias (Tabla 2). Asimismo, se determinan las estimaciones clásicas y robustas del porcentaje medio de cumplimiento de la normativa en la provincia de estudio, obteniéndose los parámetros de localización y de escala (Tabla 3 y 4 ). 
El estimador jackknife del sesgo reducido toma el valor=0,35071 (estimador insesgado). Utilizando cuatro métodos para determinar intervalos de confianza basados en las técnicas bootstrap, partiendo de la media 0,2- recortada muestral y para un coeficiente de confianza 0,95 , se obtienen valores muy aproximados (Tabla 5). Respecto a la mediana poblacional se determina el intervalo de sesgocorregido y acelerado $B C_{\alpha}=\left[0^{\prime} 25,0^{\prime} 50\right]$, e intervalo percentil [0'022239, 0'281694]. Se calcula un intervalo de confianza para la varianza obteniendo [0'11003, 0'27308] y para $\sigma$ (coeficiente de confianza 0,95 ) [18'73351, 664 '99125].

Tabla 2. Cumplimiento por "apartado"

\begin{tabular}{ccc}
\hline Apartado & $\begin{array}{c}\text { Número de edificios con equipos } \\
\text { relacionados }\end{array}$ & $\begin{array}{c}\% \\
\text { (Cumplimiento) }\end{array}$ \\
\hline I & 148 & 99,3 \\
II & 206 & 96,6 \\
III & 40 & 95 \\
IV & 55 & 30,9 \\
V & 45 & 35,5 \\
VI & 11 & 18,2 \\
VII & 4 & 0 \\
VIII & 54 & 24,1 \\
IX & 7 & 57,1 \\
X & 87 & 25,3 \\
XI & 9 & 11,1 \\
XII & 4 & 75 \\
XIII & 102 & 24,5 \\
XIV & 7 & 71,4 \\
\hline
\end{tabular}

Tabla 3. Parámetro de localización

\begin{tabular}{lcc}
\hline \multicolumn{1}{c}{ Estimador } & Valor & $\begin{array}{c}\text { Error de muestreo del } \\
\text { estimador }\end{array}$ \\
\hline Media muestral $(\bar{x})$ & 0,35 & 0,04036143 \\
Media 0'2 - Winsorizada muestral $\left(\bar{x}_{0^{\prime}}^{W}\right)$ & 0,36 & -- \\
Media 0'2 - recortada muestral $\left(\bar{x} 0_{02}^{\prime}\right)$ & 0,36 & 0,04950148 \\
Mediana muestral $\left(M_{e}\right)$ & 0,33 & 0,06019591 \\
Estimador de Huber $\left(T_{n}\right)$ & 0,36 & 0,04073724 \\
\hline
\end{tabular}

Tabla 4. Parámetro de escala

\begin{tabular}{lc}
\hline \multicolumn{1}{c}{ Estimador } & Valor \\
\hline Cuasidesviación típica muestral $(S)$ & 0,15 \\
Desviación absoluta mediana estandarizada $(N M A D)$ & 0,15 \\
Cuasidesviación típica $\quad \alpha$ - Winsorizada muestral $\left(S_{W}\right)$ & 0,11 \\
Raíz de la varianza media biponderada $\left(\hat{\xi}_{b i}^{\wedge}\right)$ & 0,15 \\
Raíz de la varianza media de porcentaje ajustado $\left(\hat{\xi}_{p a}\right)$ & 0,16 \\
\hline
\end{tabular}




\section{DISCUSIÓN}

Existe un "apartado" (ACS) no contemplado ya que para los 14 edificios "tipo" existe un cumplimiento"completo" (aprox. $100 \%$ ) de la normativa. Se estima que existe una atención especial al ACS como fuente del riesgo coincidiendo ésta con una de los sistemas más críticos. Para ella existe un cumplimiento exhaustivo y pormenorizado de los protocolos descritos en la normativa tratándose de un "apartado ejemplar" (no incluido en la Tabla 6).

Tabla 5. Intervalos de confianza bootstrap

\begin{tabular}{lc}
\hline \multicolumn{1}{c}{ Tipo de intervalo } & Valor \\
\hline Intervalo bootstrap- $t$ ideal & {$\left[0^{\prime} 268,0^{\prime} 458\right]$} \\
Intervalo bootstrap- $t$ & {$\left[0^{\prime} 270,0^{\prime} 472\right]$} \\
Intervalo percentil & {$\left[0^{\prime} 271,0^{\prime} 437\right]$} \\
Intervalo de sesgo corregido y acelerado $B C_{\alpha}$ & {$\left[0^{\prime} 284,0^{\prime} 440\right]$} \\
\hline
\end{tabular}

Para los "riesgos" en los que no aparece un único porcentaje (Tabla 6), el cero se interpreta como la falta total en la aplicación de medidas preventivas descritas en la normativa, y el que presente un valor distinto de cero es el "tipo de edificio" con menor cumplimiento (de entre los que han aplicado alguna medida, el de mayor riesgo). El objeto es la interpretación del nivel de cumplimiento, entendido éste como el de menor porcentaje; es decir, cuanto más pequeño sea un porcentaje mayor riesgo existe frente a la legionelosis ya que menos se ajusta a la normativa. La distribución del riesgo muestra el desajuste a lo descrito en la normativa nacional en su totalidad, lo que implica una mejora en los procesos preventivos. Los edificios "tipo" no representados son: Hospital, Junta de Andalucía, Hotel de 5 estrellas, Hotel Gran Lujo, Centro Público de Educación Infantil y Primaria, Centro Privado de FP, Centro Privado de Bachillerato y Centro Privado de Educación Infantil y Primaria. En ellos se produce un incumplimiento completo de la normativa ( $0 \%$ ) para los apartados señalados (Tabla 6), por esto no se representan. Hay que considerar que en la estimación del "mayor riesgo" estos edificios "tipo" son los que presentan el valor más alto, con un menor porcentaje. Se deduce que no existen edificios "tipo" que no hayan sido contemplados (Tabla 6), ya que o han realizado medidas preventivas insuficientes o no han implantado ninguna.

Respecto al "mayor riesgo" cabe especial atención, en el seguimiento del cumplimiento de la normativa, los edificios turísticos o sector hotelero, ya que en ellos se centra el mayor incumplimiento parcial. Resaltar la existencia de "tipos de edificios" como son los hospitales, tan relevantes desde el punto de vista sanitario y de enorme impacto potencial en la población, en los que para el estudio definido se marcan en algunos riesgos (humectación en conductos de aire, 
líneas de agua en unidades dentales) una ausencia en la adopción de medidas preventivas. Al mismo tiempo y con la salvedad de los edificios más críticos, se observa como hay edificios "tipo" que presentan varios apartados, algunos con el mismo valor y otros no. Se diferencia como determinados apartados ("mayor riesgo") únicamente están "asociados" con un "tipo de edificio". Hay que considerar que los edificios incluyen personas de distintas edades y características, desde niños hasta individuos de edad avanzada, pudiendo el riesgo resultar aún mayor.

Tabla 6. Tipo de edificio/mayor riesgo

\begin{tabular}{|c|c|c|}
\hline "Tipo de edificio" & $\begin{array}{c}\text { Mayor riesgo } \\
\text { (apartado, resumido) }\end{array}$ & Porcentaje \\
\hline Instituto de Educación Secundar ia (público) & AFS & 85,71 \\
\hline Hotel 4 estrellas & TORRES & 81,81 \\
\hline Hotel 3 estrellas & BAN̈ERAS & 21,42 \\
\hline $\begin{array}{l}\text { Instituto de Educación Secundaria (público), Centro } \\
\text { Público de Educación Inf. y Primaria, Centro Privado de } \\
\text { Educación Inf. y Primaria y Centro Privado de ESO }\end{array}$ & BAÑERAS & 0,00 \\
\hline Hotel 4 estrellas & PISCINAS & 29,41 \\
\hline $\begin{array}{l}\text { Centro Privado de Bachillerato, Centro Privado de } \\
\text { Educación Inf. y Primaria y Centro Privado de ESO }\end{array}$ & PISCINAS & 0,00 \\
\hline $\begin{array}{l}\text { Administración (no Junta de Andalucía) y Hotel } 3 \\
\text { estrellas }\end{array}$ & HUMECTACIÓN & 50,00 \\
\hline $\begin{array}{l}\text { Hospital, Hotel } 4 \text { estr ellas, Hotel } 5 \text { estrellas y Hotel Gran } \\
\text { Lujo }\end{array}$ & HUMECTACIÓN & 0,00 \\
\hline Hospital y Centro Público de Educación Inf. y Primaria & DENTALES & 0,00 \\
\hline Hotel 2 estrellas & FUENTES & 16,66 \\
\hline $\begin{array}{l}\text { Administración (no Junta de Andalucia), Junta de } \\
\text { Andalucia, Instituto de Educación Secundari a (público), } \\
\text { Centro Público de Educación inf. y Primaria, Centro } \\
\text { Privado FP y Centro Privado de ESO }\end{array}$ & FUENTES & 0,00 \\
\hline $\begin{array}{l}\text { Administración (no Junta de Andalucía) y Hotel } 3 \\
\text { estrellas }\end{array}$ & CONDUCTO & 50,00 \\
\hline Centro Privado de ESO & RIEGO & 11,11 \\
\hline $\begin{array}{l}\text { Junta de Andalucia, Instituto de Educación Secundaria } \\
\text { (público), Centro Público de Educación Inf. y Primaria, } \\
\text { Centro Privado FP y Centro Privado de Educación Inf. y } \\
\text { Primaria }\end{array}$ & RIEGO & 0,00 \\
\hline $\begin{array}{l}\text { Hotel } 4 \text { estrellas, Hotel } 5 \text { estrellas y Centro Público de } \\
\text { Educación Inf. y Primaria }\end{array}$ & TERMALES & 0,00 \\
\hline Hotel 4 estrellas & ACUARIOS & 50,00 \\
\hline Hotel 2 estrellas & $\mathrm{SCl}$ & 20,00 \\
\hline $\begin{array}{l}\text { Instituto de Educación Secundaria (público), Centro } \\
\text { Público de Educación Inf. y Primaria, Centro Privado FP } \\
\text { y Centro Privado de Educación Inf. y Primaria y Centro } \\
\text { Privado de ESO }\end{array}$ & $\mathrm{SCl}$ & 0,00 \\
\hline Hotel 3 e strellas & TERAPIA & 0,00 \\
\hline
\end{tabular}

No existe una diferencia relevante en si un edificio es de gestión pública o privada y el incumplimiento de la normativa. Para los edificios "tipo" con alguna medida preventiva insuficiente, es conveniente resaltar que el mayor riesgo se 
presenta en los edificios que no son de la Administración Pública. Los edificios de gestión privada y en especial los hoteles es donde más riesgo existe, aumentando éste cuanta menor categoría tenga el hotel. Precisar que en los edificios de la Administración (no pertenecientes a la Junta de Andalucía) y Hoteles de 3 estrellas, el porcentaje de "mayor riesgo" es del $50 \%$ aunque puntualizando que le corresponden dos riesgos con el mismo porcentaje.

En la búsqueda del estimador asintóticamente más eficiente dentro de los que tienen acotada su sensibilidad a grandes errores se propone el estimador B- robusto óptimo y, en el caso de los $M$ - estimadores de localización es el estimador de Huber y para los $M$ - estimadores de escala la desviación absoluta mediana estandarizada $N M A D^{1}$. Por ello, resulta interesante elegir no sólo un estimador robusto, sino que tenga sesgo reducido. De esta forma se sugiere como estimador de localización el de Huber o la media $\alpha$ - recortada muestral (con $\alpha=0$ '2); y como estimador de escala, la raíz cuadrada de la varianza media biponderada o $N M A D$, ya que todos estos estimadores tienen un error de muestreo sólo algo mayor que la media muestral o la cuasidesviación típica muestral, cuando la distribución modelo es normal, y mantienen esos errores de muestreo casi invariables aún con distribución modelo no normal. No se presentan grandes diferencias en los valores ni siquiera en el caso de $S$ al no existir asimetría en los datos. Como estimadores de escala recomendados se presentan $\hat{\xi}_{b i}$ y $N M A D$ (más insensibles), concluyendo con $N M A D=0,15$ como estimación final; y de localización la media recortada y el estimador de posición de Huber $=0,36$ concluyendo con éste último.

El hecho de que de 214 edificios haya 130 que no "respeten" la normativa es un indicador de la falta de rigor existente en la materia y aún más del enorme riesgo biológico (Legionella pneumophila) presente en la provincia de Málaga. En este sentido, se estima sumamente importante un mayor seguimiento del cumplimiento, bien por parte de la Administración (labores de inspección) bien por empresas contratadas al efecto que sirvan de apoyo en este tipo de tareas.

Según el p-valor se rechaza la hipótesis nula de independencia entre las variables "tipo de edificio" y cumplimiento de la normativa. Las estimaciones del parámetro de localización cumplimiento medio, llevan a concluir que la estimación razonable de dicho cumplimiento es la de 0,36 (36\%). En la provincia de

\footnotetext{
${ }^{1}$ Hampel FR. [Contributions to the theory of robust estimation] Tesis doctoral [1968]. Se localiza en Universidad de California, Berkeley.
} 
Málaga (España) y para los edificios muestreados es pertinente una mayor implementación en las herramientas de control de la legionelosis y en la aplicación de los métodos de control para un correcto mantenimiento higiénico-preventivo

Agradecimientos. Este estudio ha sido parcialmente financiado mediante una ayuda de investigación de la Dirección General de Seguridad y Salud Laboral (Consejería deEmpleo) de la Junta de Andalucía (España).

\section{REFERENCIAS}

1. Smith TJ. Exposure assessment for occupational epidemiology. Am J Ind Med. 1987;12: 249-268. 2. Szewzyk U, Szewzyk R, Manz W, Schleifer KH. Microbiological safety of drinking water. Annu Rev Microbiol. 2000; 54: 81-127.

3. Atlas RM. Legionella: from environmental habitats to disease pathology, detection and control. Environ Microbiol. 1999 Aug; 1(4): 283-293.

4. Saint CP, Ho L. A PCR test for the identification and discrimination of Legionella longbeachae serogroups 1 and 2. J Microbiol Methods. 1999 Sep; 37(3): 245-253.

5. Kasuga O, Takagi K, Tani K, Kinumaki A. Studies on detection methods for Legionella species from environmental water. Kansenshogaku- Zasshi. 1999 Jan; 73(1): 25-34.

6. Ruckdeschel G, Dalhoff A. The in-vitro activity of moxifloxacin against Legionella species and the effects of medium on susceptibility test results. J Antimicrob Chemother. 1999 May; 43 Suppl B: 25-29.

7. Brown CM, Nuorti PJ, Breiman RF, Hathcock AL, Fields BS, Lipman HB et al. A community outbreak of legionnaires' disease linked to hospital cooling towers: an epidemiological method to calculate dose of exposure. Int J Epidemiol. 1999 Apr; 28(2): 353-359.

8. Kelly AA, Danko LH, Kralovic SM, Simbartl LA, Roselle GA. Legionella in the veterans healthcare system: report of an eight-year survey. Epidemiology and Infection. 2003 Oct; 131 (2): 835-839.

9. Boletín Oficial del Estado. Real Decreto $865 / 2003$, de 4 de julio, por el que se establecen los criterios higiénico- sanitarios para la prevención y control de la legionelosis. BOE núm $171,18 / 7 / 2003$.

10. Boletín Oficial de la Junta de Andalucía. Decreto 287/2002, de 26 de noviembre, por el que se establecen medidas para el control y la vigilancia higiénico- sanitarias de instalaciones de riesgo en la transmisión de la legionelosis y se crea el Registro Oficial de Establecimientos y Servicios Biocidas de Andalucía. BOJA núm 144, 7/12/2002. 